\title{
Multiple sclerosis: Implications for future research on postural control and gait
}

\author{
FELIPE B. SANTINELLI ${ }^{1}$ | EMERSON SEBASTIÃO² | MARCELA DE OLIVEIRA ${ }^{3}$ FABIO A. BARBIERI ${ }^{1}$
}

\author{
1 São Paulo State University (UNESP) - Campus Bauru, School of Science, Human Movement Research Laboratory (MOVI-LAB), Department of Physical Education, \\ Bauru-SP, Brazil. \\ ${ }^{2}$ Department of Kinesiology and Physical Education, Northern Illinois University, United States. \\ ${ }^{3}$ São Paulo State University (UNESP) - Campus Bauru, School of Science, Advanced Materials and Nanoparticles Laboratory, Physics Department, Bauru-SP, Brazil. \\ Correspondence to: Fabio Augusto Barbieri. Corresponding author at São Paulo State University (Unesp) - Campus Bauru, School of Science, Human Movement \\ Research Laboratory (MOVI-LAB), Department of Physical Education, Bauru-SP, Brazil. \\ email: fabio.barbieri@unesp.br \\ https://doi.org/10.20338/bjmb.v14i2.169
}

$\begin{array}{ll}\text { ABBREVIATIONS } \\ \text { CNS } \quad \text { central nervous system } \\ \text { EDSS } & \text { Expanded disability Status Scale } \\ \text { MS } & \text { Multiple Sclerosis }\end{array}$

PUBLICATION DATA

Received 11042020

Accepted 29052020

Published 01072020

\begin{abstract}
This letter to the editor has the objective of providing a perspective of the impact of multiple sclerosis (MS) on postural control and gait, the current studies, and suggestions for future studies. Although studies on MS with postural control and gait have been carried out for some time, in Brazil and in the World, there is still insufficient information on MS and impairments in postural control and gait. Postural control and gait impairments are recognized to cause several problems for people with MS, these being two of the symptoms that most affect quality of life. Here, we present studies that have investigated impairments in postural control and gait using different experimental designs and discuss the adaptations of the central nervous system (CNS) due to the damage caused by MS. We recommend future studies focus on how the CNS is organized towards postural control and gait, with a better ecological approach, which could assist the development of rehabilitation programs.
\end{abstract}

KEYWORDS: Multiple Sclerosis | Postural control | Gait | Motor Control | Movement | Central nervous system

\section{INTRODUCTION}

Multiple Sclerosis (MS) is a neurodegenerative, often disabling, disease of the central nervous system (CNS) that mostly affects young adults around the world. The diagnosis of MS is complex, given the fact that symptoms are similar to numerous other conditions. MS is characterized by the demyelization process in the neurons of the CNS. Recent findings suggest that brain atrophy in MS occurs at a faster rate (1\% per year) compared to healthy people $\left(0.1-0.3 \%\right.$ per year). ${ }^{1,2}$ Thus, several symptoms are presented by this population, such as impairments in bladder function, increased fatigue, loss of coordination, cognitive decline, muscular weakness, vision problems (i.e. double vision or vision loss), and impairments in postural control and gait. ${ }^{3}$ Postural control and gait are essential for independence in people with MS, and impairment in those aspects negatively affects quality of life in this population. Although MS has been recognized since early in the 19th century, knowledge and research on MS are incipient, especially in Brazil. The effects of MS on movement, especially related to gait and posture, are relatively new and controversy. To this end, the focus of this letter is to provide interpretations on how MS affects both postural control and gait.

Postural control requires interactions between the subject with the environment and task, for example, remaining in an upright stance. Information on these factors is obtained through visual, vestibular, and somatosensory systems, as well as the muscular system, through the muscle spindle and Golgi tendon organ, which could be affected in people with MS, leading to impairments in postural control. ${ }^{4}$ In this view, previous 
studies/works suggested that the impairments may be dependent on the level of disability. ${ }^{5}$ In fact, higher levels of disability (demonstrated by the Expanded disability Status ScaleEDSS) have been associated with low brain volume, low grey and white matter, and higher lesion load6; which was related to higher body sway (i.e. higher amplitude and displacement of the center of pressure). ${ }^{5}$ Moreover, the association of a secondary task may be an important aspect in the maintenance of balance, which could improve or decrease postural control performance. For example, when manipulating the task (e.g. saccadic eye movement), people with MS with mildly disability are able to reduce body sway (i.e. lower amplitude and displacement of center of pressure), ${ }^{7}$ while the addition of a cognitive task (e.g. dual task), negatively influenced postural control. ${ }^{8}$ Therefore, impairments in postural control in people with MS seem to be postural task-related, which is highlighted in greater levels of disability. ${ }^{9}$

As well as postural control, gait requires interactions of several types of information, such as proprioception (obtained through somatosensory and vestibular system) and exteroception (obtained through visual system), for efficient performance. Contrary to what is observed in postural control, gait abnormalities are seen even in early stages of the disease. ${ }^{10}$ People with MS often present shorter step length, slower step velocity, longer step duration and double support time, and greater step width when compared with neurologically healthy individuals. ${ }^{11,12}$ Rocca et al. ${ }^{13}$ demonstrated that in people with MS with damage to motor pathways, mainly in the pyramidal tract, upper-right limb movement is impaired, requiring more cortical resource to accomplish this motor task. Indeed, people with MS with dysfunction of the pyramidal system presented worse performance in gait parameters when compared with their counterparts with impairments in the sensory or cerebellar system, ${ }^{14}$ which was also dependent on the level of disability. ${ }^{11}$ In addition, people with MS seem to require more cortical resource to accomplish gait with dual task than controls. ${ }^{15} \mathrm{MS}$ appears to have a significant negative impact on gait. ${ }^{16}$ Recent findings suggest a change in CNS activation/organization (i.e. how the CNS receives the sensory information, organizes this information, plans the response, and responds according to the environment and task demands), ${ }^{15,17}$ even in the early stages, and related to the level of disability.

In conclusion, MS affects both postural control and gait. The impairments seem to be disability-dependent; meaning that the higher the level of disability, the higher the level of impairments. Interestingly, people with MS in the early stages of the disease appear to demonstrate no (or little) impairments in postural control. It is possible that postural control may be an easier task. Based on recent studies and higher heterogeneity of MS, defining a common explanation of the main cause of impairments in postural control and gait is not an easy mission. However, both the demyelization process and brain atrophy, ${ }^{18}$ mainly in motor pathways, ${ }^{13}$ which is more evident in higher levels of disease, seem to be reasonable explanations for the motor impairments suffered by this population. To the best of our knowledge, the majority of the studies that investigated movement and CNS activation/organization in MS, used $\mathrm{PMRI}$ equipment, ${ }^{13}$ which decreases the ecological approach. Although recent studies have investigated CNS activation/organization through real situations, there is a gap in the literature in understanding how people with MS deal with the damage in the CNS.

Future studies with MS should more deeply explore how the CNS adjusts or compensates for the damage due to MS, to accomplish gait and postural control, which could provide a more detailed explanation about the impairments in these aspects in MS. 
For example, investigating the CNS during a challenging motor task, such as gait and postural control with motor or cognitive dual tasking, could increase the ecological validity and help to understand brain functioning. Recently, some studies with fMRI investigated how the CNS behaves during gait in people with MS; however, with the use of an imaginary task, which significantly reduced ecological validity. Thus, it is important to find an alternative way to evaluate brain activity, which is equivalent to the data observed in fMRI but with better ecological validity. This better ecological validity could help us to understand the consequences of MS on postural control and gait, which may lead to improvement in the protocols applied in rehabilitation programs for people with MS.

\section{ACKNOWLEDGMENTS}

This work was supported by Fundação de Amparo a Pesquisa do Estado de São Paulo (FAPESP) [grant number 2018/18078-0 and 2017/20032-5] and in part by the Coordenação de Aperfeiçoamento de Pessoal de Nível Superior (Code 001).

\section{REFERENCES}

1. Barkhof F, Calabresi PA, Miller DH, Reingold SC. Imaging outcomes for neuroprotection and repair in multiple sclerosis trials. Nat Rev Neurol. 2009;5(5):256-266. doi:10.1038/nrneurol.2009.41.

2. Trapp BD, Vignos M, Dudman J et al. Cortical neuronal densities and cerebral white matter demyelination in multiple sclerosis : a retrospective study. Lancet Neurol. 2018;17(10):870884 .

3. Thompson AJ, Baranzini SE, Geurts J, Hemmer B, Ciccarelli O. Multiple sclerosis. Lancet. 2018;391(10130):1622-1636. doi:10.1016/S0140-6736(18)30481-1.

4. Cameron MH, Nilsagard Y. Balance, gait, and falls in multiple sclerosis. Handb Clin Neurol. 2018;159:237-250. doi:10.1016/B978-0-444-63916-5.00015-X.

5. Pau M, Porta M, Coghe $G$ et al. Are static and functional balance abilities related in individuals with Multiple Sclerosis? Mult Scler Relat Disord. 2017;15(February):1-6. doi:10.1016/j.msard.2017.04.002.

6. Dimitrov I, Georgiev R, Kaprelyan A et al. Brain and Lesion Volumes Correlate With Edss in Relapsing-Remitting Multiple Sclerosis. J of IMAB. 2015;21(4):1015-1018. doi:10.5272/jimab.2015214.1015.

7. Santinelli FB, van Emmerik REAA, Silva FA et al. Saccadic eye movements are able to reduce body sway in mildly-affected people with Multiple Sclerosis. Mult Scler Relat Disord. 2019;30(August 2018):63-69. doi:10.1016/j.msard.2019.02.005.

8. Boes MK, Sosnoff JJ, Socie MJ, Sandroff BM, Pula JH, Motl RW. Postural control in multiple sclerosis: Effects of disability status and dual task. J Neurol Sci. 2012;315(1-2):4448. doi:10.1016/j.jns.2011.12.006.

9. Comber L, Sosnoff JJ, Galvin R, Coote S. Postural control deficits in people with Multiple Sclerosis: A systematic review and meta-analysis. Gait Posture. 2018;61(October 2017):445-452. doi:10.1016/j.gaitpost.2018.02.018. 
10. Martin CL, Phillips BA, Kilpatrick TJ et al. Gait and balance impairment in early multiple sclerosisInin the absence of clinical disability. Mult Scler. 2006;12(5):620-628. doi:10.1177/1352458506070658.

11. Preiningerova JL, Novotna K, Rusz J, Sucha L, Ruzicka E, Havrdova E. Spatial and temporal characteristics of Gait as outcome measures in multiple sclerosis (EDSS 0 to 6.5). J Neuroeng Rehabil. 2015;12(1):1-7. doi:10.1186/s12984-015-0001-0.

12. Gianfrancesco MA, Triche EW, Fawcett JA, Labas MP, Patterson TS, Lo AC. Speed- and cane-related alterations in gait parameters in individuals with multiple sclerosis. Gait Posture. 2011;33(1):140-142. doi:10.1016/j.gaitpost.2010.09.016.

13. Peterson DS, Fling BW. How changes in brain activity and connectivity are associated with motor performance in people with MS. Neurolmage Clin. 2018;17(September 2017):153162. doi:10.1016/j.nicl.2017.09.019.

14. Kalron A, Givon U. Gait characteristics according to pyramidal, sensory and cerebellar EDSS subcategories in people with multiple sclerosis. J Neurol. 2016;263(9):1796-1801. doi:10.1007/s00415-016-8200-6.

15. Chaparro G, Balto JM, Sandroff BM et al. Frontal brain activation changes due to dualtasking under partial body weight support conditions in older adults with multiple sclerosis. J Neuroeng Rehabil. 2017;14(1):1-10. doi:10.1186/s12984-017-0280-8.

16. Comber L, Galvin R, Coote S. Gait deficits in people with multiple sclerosis: A systematic review and meta-analysis. Gait Posture. 2016;51:25-35. doi:10.1016/j.gaitpost.2016.09.026.

17. Hernandez ME, Holtzer R, Chaparro $G$ et al. Brain activation changes during locomotion in middle-aged to older adults with multiple sclerosis. J Neurol Sci. 2016;370:277-283. doi:10.1016/j.jns.2016.10.002.

18. van Munster CEP, Jonkman LE, Weinstein HC, Uitdehaag BM, Geurts JJ. Gray matter damage in multiple sclerosis: Impact on clinical symptoms. Neuroscience. 2015;303:446461. doi:10.1016/j.neuroscience.2015.07.006.

Citation: Santinelli FB, Sebastião E, de Oliveira M, Barbieri FA. Multiple sclerosis: Implications for future research on postural control and gait. BJMB. 2020: 14(2): 46-49.

Editors: Dr José Angelo Barela - São Paulo State University (UNESP), Rio Claro, SP, Brazil; Dr Natalia Madalena Rinaldi - Federal University of Espírito Santo (UFES), Vitória, ES, Brazil.

Copyright:@ 2020 Santinelli, Sebastião, de Oliveira and Barbieri and BJMB. This is an open-access article distributed under the terms of the Creative Commons Attribution-NonCommercial-NoDerivatives 4.0 International License which permits unrestricted use, distribution, and reproduction in any medium, provided the original author and source are credited.

Funding: This work was supported by Fundação de Amparo a Pesquisa do Estado de São Paulo (FAPESP) [grant number 2018/18078-0 and 2017/20032-5] and in part by the Coordenação de Aperfeiçoamento de Pessoal de Nível Superior (Code 001).

Competing interests: The authors have declared that no competing interests exist. DOl:https://doi.org/10.20338/bjmb.v14i2.169 